\title{
EEG Beta Wave Trains Are Not the Second Harmonic of Mu Wave Trains in Parkinson's Disease Patients
}

\author{
Olga S. Sushkova ${ }^{1}$, Alexei A. Morozov ${ }^{1,2}$, Alexandra V. Gabova ${ }^{3}$ \\ ${ }^{1}$ Kotel'nikov Institute of Radio Engineering and Electronics of RAS, Mokhovaya 11-7, Moscow, 125009, Russia \\ ${ }^{2}$ Moscow State University of Psychology E Education, Sretenka 29, Moscow, 107045, Russia \\ ${ }^{3}$ Institute of Higher Nervous Activity and Neurophysiology of RAS, Butlerova 5A, Moscow, 117485, Russia
}

\begin{abstract}
The goal of this study is development of a novel signal processing and analysis method for detailed investigation of the time-frequency dynamics of brain cortex electrical activity. The idea of our method of electroencephalograms (EEG) analyzing is in that we consider EEG signal as a composition of so-called wave trains. The wave train term is used to denote a signal localized in time, frequency, and space. We consider the wave train as a typical component of EEG, but not as a special kind of EEG signals.

In contrast to papers devoted to detecting wave trains of one or two specific types, such as alpha spindles and sleep spindles, we analyze any kind of wave trains in a wide frequency band. Using this method, we have found three interesting frequency areas where differences were detected between a group of Parkinson's disease (PD) patients and a control group of healthy volunteers. The goal of this work is to check whether the regularities in the mu and beta frequency bands are independent ones, that is, the beta wave trains observed in the analysis were not the second harmonics of the mu wave trains.

We have developed a special algorithm that eliminates from the analysis all beta wave trains in EEG signal that were observed simultaneously with the mu wave trains. Analysis of a real experimental data set processed by this algorithm has confirmed that the beta frequency band regularity is separate from the mu frequency band regularity. Moreover, a new significant difference between the left hand tremor and right hand tremor Parkinson's disease patients was discovered.
\end{abstract}

Keywords: Wave train, Wave packet, Burst, Electroencephalogram, EEG, Beta, Mu, Wavelet, Visualizing EEG data, Decrease of quantity of wave trains, Parkinson's disease

\section{Introduction}

The goal of our research is development of a novel signal processing and analysis method for detailed investigation of time-frequency dynamics of brain cortex electrical activity. The idea of our method of analyzing EEG is in that we consider EEG signal as a composition of so-called wave trains. In contract to papers devoted to detecting wave trains of one or two specific types, such as alpha spindles [1] and sleep spindles [2, 3, 4, 5, 6, 7], we analyze any kind of wave trains in a wide frequency area. The developed method differs from analogous method for detailed analysis of time-frequency dynamics of EEG [8] in that the statistical analysis of samples of wave trains and a new method for visualizing the results of the analysis are proposed. The algorithm used for detecting wave trains also is different. In particular, a new kind of diagrams based on ROC curves was developed to visualize the neurophysiological data (see an example of the diagram in Figure 6, Section 2).

In physics, a wave train (or a wave packet) is a short "burst" or "envelope" of localized wave action that travels as a unit. In this paper, the wave train term is used to denote a signal localized in time, frequency, and space. We consider the wave train as a typical pattern in EEG signals. Recently we have demonstrated that the number of wave trains in the EEG beta frequency range $(12-25 \mathrm{~Hz})$ is significantly decreased in early stage Parkinson's disease patients $[9,10]$. In previous paper [11], a method of visualization of EEG analysis results based on ROC curves was described. Using this method, we have revealed three interesting frequency ranges where differences between a Parkinson's disease patient group and a control group are detected. The first range is $7.5-9.5 \mathrm{~Hz}$ (approximately the mu frequency band), 
the second is $10.5-13.5 \mathrm{~Hz}$ (also the mu frequency band), and the third range is $18-24 \mathrm{~Hz}$ (approximately the beta- 2 frequency band). The presence of the first and second frequency bands gives an evidence for a shift of EEG mu rhythm to the lower frequency areas in Parkinson's disease patients. The third frequency band is a confirmation of the regularity reported in [9]. Note that in [9] another frequency band was investigated $(12-25 \mathrm{~Hz})$. As a consequence, the numerical characteristics of EEG given in this paper differ slightly from the numerical characteristics considered in the paper [9]. In addition, the frequency ranges of beta-1 and beta- 2 were not separated in the paper [9], but in the course of further research we came to the conclusion that the characteristics of the wave trains in these beta sub-bands should be investigated separately.

The goal of this work is to check whether the regularities in the mu and beta frequency bands are independent ones, that is, the beta wave trains observed in the analysis were not the second harmonics of the mu wave trains. This check is important for understanding the development of neurodegenerative processes and the formation of compensatory neurophysiological mechanisms in Parkinson's disease, since the frequency ranges of mu and beta correspond to the work of different functional systems in the brain $[12,13,14,15])$. We have developed a special algorithm that eliminates from the analysis all beta wave trains in EEG signal that were observed simultaneously with the mu wave trains. Analysis of a real experimental data set processed by this algorithm has confirmed that the beta frequency band regularity is separate from the mu frequency band regularity. Furthermore, a new significant difference between the left hand tremor and right hand tremor Parkinson's disease patients was discovered.

\section{A Problem Statement}

Let $M$ be a local maximum in a wavelet spectrogram (see Figure 1). We estimate the full width at half maximum (FWHM) of $M$ in the time plane $F W H M_{\text {TIME }}$ and in the frequency plane $F W H M_{F R E Q U E N C Y}$. Then we check whether
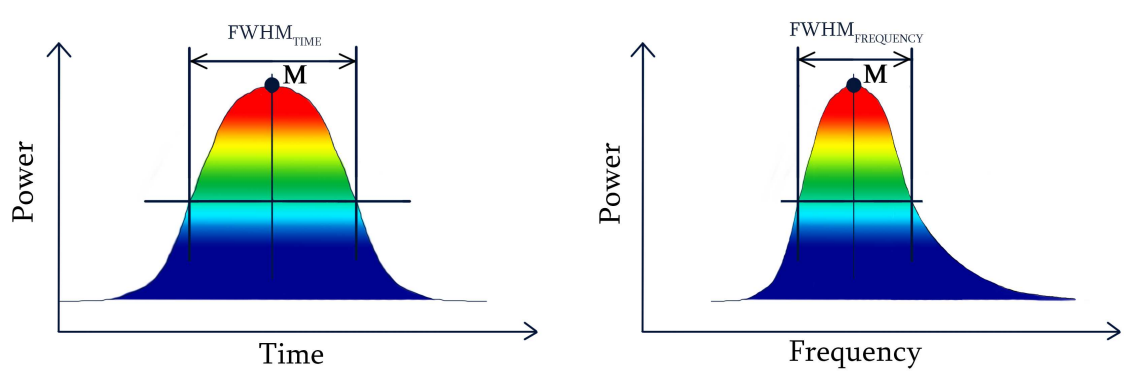

Figure 1: An example of a spectrogram of a wave train in a time-frequency domain. The diagram at the left shows the spectrogram of the signal in the time plane. The abscissa indicates a time and the ordinate indicates a power. The diagram at the right shows the spectrogram of the signal in the frequency plane. The abscissa indicates a frequency and the ordinate indicates a power.

there are no values in the rectangle area

\section{$F W H M_{T I M E} \times F W H M_{F R E Q U E N C Y}$}

that are bigger than the $M$ value (see Figure 2). We consider $M$ as a case of a wave train if $F W H M_{T I M E}$ of $M$ is greater or equal to the $T_{D}$ threshold (see Figure 1). The $T_{D}$ threshold is a function of the frequency $f$ of the maximum $M:$

$$
F W H M_{T I M E} \geq T_{D}=N_{P} / f
$$

where $N_{P}$ is a constant given by an expert. In this paper, we apply the value: $N_{P}=2$.

An example of wave trains in the time-frequency domain in a PD patient is shown in Figure 3. The figure demonstrates the wave trains in the background EEG in the right hand tremor patient, the C3 cortex area. Each circle indicates a wave train in the wavelet spectrogram. It is obvious that most wave trains are located in the mu frequency band $(8-12 \mathrm{~Hz})$. In addition, there are wave trains in the delta $(1-4 \mathrm{~Hz})$, theta $(4-8 \mathrm{~Hz})$, and beta $(12-30 \mathrm{~Hz})$ frequency bands. 


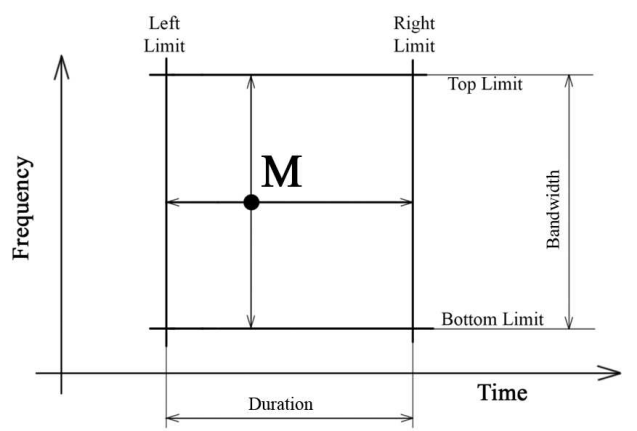

Figure 2: Time and frequency bounds of the $M$ wave train in the wavelet spectrogram. The abscissa indicates the time and the ordinate indicates the frequency.

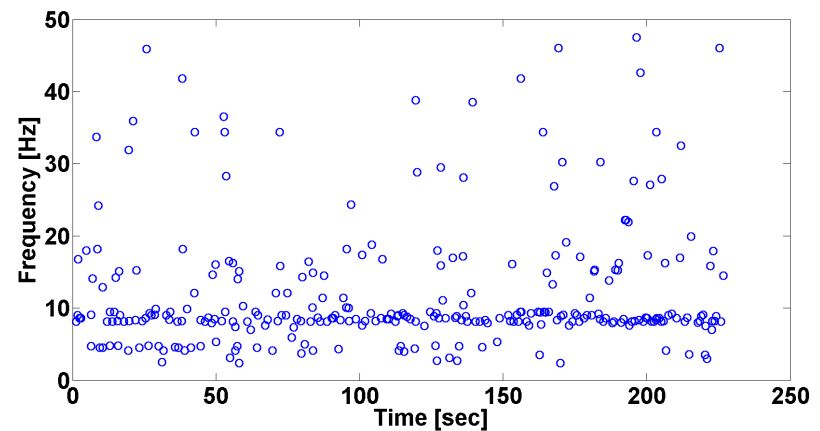

Figure 3: Wave trains of the PD patient in the time-frequency domain. A background EEG in the right hand tremor patient is presented, the C3 cortex area, the delta $(1-4 \mathrm{~Hz})$, theta $(4-8 \mathrm{~Hz}), \mathrm{mu}(8-12 \mathrm{~Hz})$, and beta $(12-30 \mathrm{~Hz})$ frequency bands. Each circle indicates a wave train in the wavelet spectrogram. The abscissa is the time and the ordinate is the frequency.
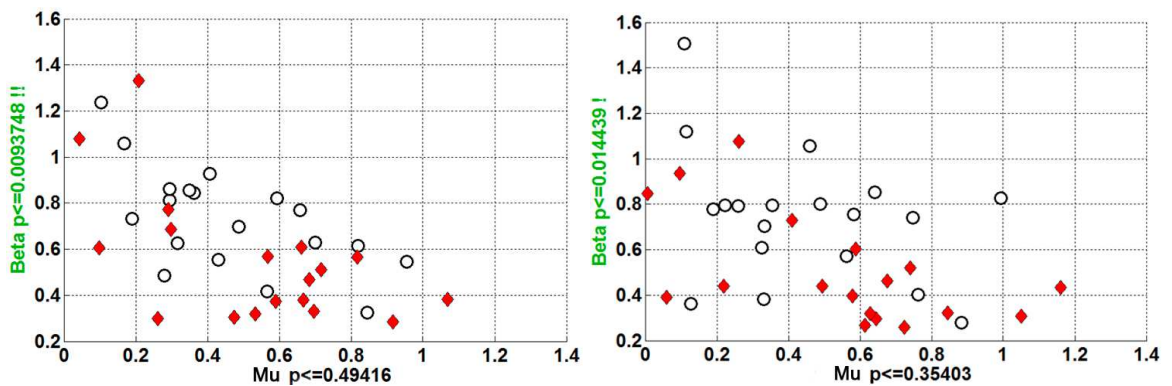

Figure 4: The scattering of the quantity of beta wave trains in the patients and the healthy volunteers. The C3 cortex area is shown at the left, the $\mathrm{C} 4$ cortex area is shown at the right. The abscissa is the quantity of wave trains per second in the mu frequency band. The ordinate is the quantity of wave trains per second in the beta frequency band. The patients are indicated by diamonds and the healthy volunteers are indicated by circles.

The method of EEG wave train analysis revealed a new effect in the Parkinson's disease [9, 10]. The number of beta $(12-25 \mathrm{~Hz})$ wave trains in the C3 and C4 cortex areas is significantly decreased (Mann-Whitney, $p<0.02)$, see Figure 4. The patients are indicated by red diamonds, and the healthy volunteers are indicated by white circles. Each diamond and each circle correspond to a particular person. The number of wave trains is standardized by the length of EEG record in seconds, because durations of EEG records were slightly different in the subjects. Note that in this test the quantity of the wave trains is considered only, but not the amplitude of the wave trains.

In previous paper [11], a method of visualization of results of EEG analysis based on ROC curves was described. Let MinFreq, MaxFreq are frequency bounds of a four-dimensional area $S$ in the space of the wave trains. Let 
MinPower, MaxPower are power bounds of the area $S$; MinDurat, MaxDurat are duration bounds of the area $S$; and MinBandwidth, MaxBandwidth are bandwidth bounds of the area $S$. It was calculated a number of wave trains per second located in the area $S$ in every individual patient and healthy volunteer and created histograms of the quantity of the wave trains per second (see an example in Figure 5). A statistical difference between the diagrams may indicate that the area $S$ contains wave trains that are typical for Parkinson's disease patients, but not for the control group, or vice versa. Another interesting issue is whether it can be specified a threshold (a limit of the number of the wave trains in the area $S$ ) that separates adequately the histograms, because the presence of such threshold means that the quantity of the wave trains in the area $S$ may be used for the clinical diagnosis of Parkinson's disease. For instance, there is a strong statistical difference between the histograms in the Figure 5 (the Mann-Whitney test, $p<0.009$ ). The diagram demonstrates that a typical number of the wave trains in the control group is about 0.13 per second in the given frequency band. At the same time, a typical number of the wave trains in the patients is about 0.06 .
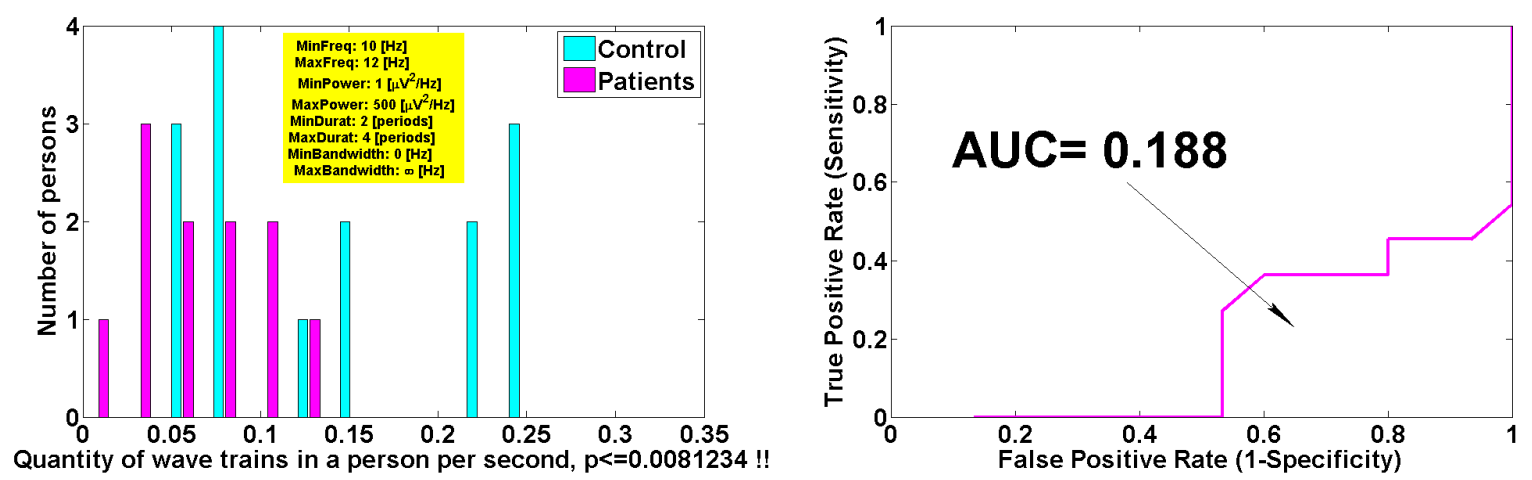

Figure 5: At the left: histograms of the quantity of the wave trains per second in the patients and the control group (the left hand tremor patients, the $\mathrm{C} 3$ cortex area). The wave trains are considered in the $S$ space bounded by the following limits: a frequency range is $10-12 \mathrm{~Hz}$, a power range is $1-500 \mu V^{2} / H z$, the duration range is $2-4$ periods, the bandwidth range has no limits. The patients' histogram is indicated by the dark magenta color; and the control group histogram is indicated by the light cyan color. At the right: a ROC curve based on the histograms. The abscissa indicates the False Positive Rate. The ordinate indicates the True Positive Rate. The area under the ROC curve $(A U C)$ indicates whether the area $S$ is applicable for separation of the patients and the control group. $A U C<0.5$ indicates that the wave trains quantity is greater in the control group than in the patients.

Thus, in mathematical terms, the goal of our investigation was searching such areas in the multidimensional space of the wave trains, where $A U C$ differs sufficiently from 0.5 and is approached to 1 or to 0 . AUC $>0.5$ indicates that the wave trains quantity is greater in the patients than in the healthy volunteers. Similarly, $A U C<0.5$ indicates that the wave trains quantity is greater in the control group. An exhaustive search of the values MinFreq, MaxFreq, MinPower, MaxPower, MinDurat, MaxDurat, MinBandwidth, and MaxBandwidth can be implemented to investigate the multidimensional space, but we consider different slices of the space using various $2 \mathrm{D}$ and $3 \mathrm{D}$ diagrams not to miss any interesting regularities in the space of the wave trains. An example of this analysis is presented in the Figure 6.

Let us compute $A U C$ values for various frequency ranges. In Figure 6, the functional dependence of $A U C$ is shown, where the arguments of the function are the MinFreq and MaxFreq bounds. The frequency values varied from 2 to $25 \mathrm{~Hz}$ (with the $0.5 \mathrm{~Hz}$ step); the MinPower, MaxPower, MinDurat, MaxDurat, MinBandwidth, and MaxBandwidth were constant: MinPower $=1$, MaxPower $=\infty$, MinDurat $=0$, MaxDurat $=\infty$, MinBandwidth $=$ 0, MaxBandwidth $=\infty$. The upper left triangle of the diagram indicates the values of $A U C$ corresponding to the MinFreq-MaxFreq frequency range. The lower right triangle of the diagram indicates the $A U C$ values corresponding to the total frequency band $2-25 \mathrm{~Hz}$ except the MaxFreq-MinFreq band. Note that in the lower right triangle of the diagram the MinFreq indicates the upper limit (but not the lower limit) of the excepted frequency band and the MaxFreq indicates the lower bound of the excepted values.

Using this method we have revealed three interesting frequency ranges where differences between a Parkinson's disease patient group and a control group were detected [11]. The first range is $7.5-9.5 \mathrm{~Hz}$ (approximately the $\mathrm{mu}$ frequency band), the second is $10.5-13.5 \mathrm{~Hz}$ (also the mu frequency band), and the third range is $18-24 \mathrm{~Hz}$ (approximately the beta-2 frequency band). The task of this paper is to check whether the regularities in EEG in the 


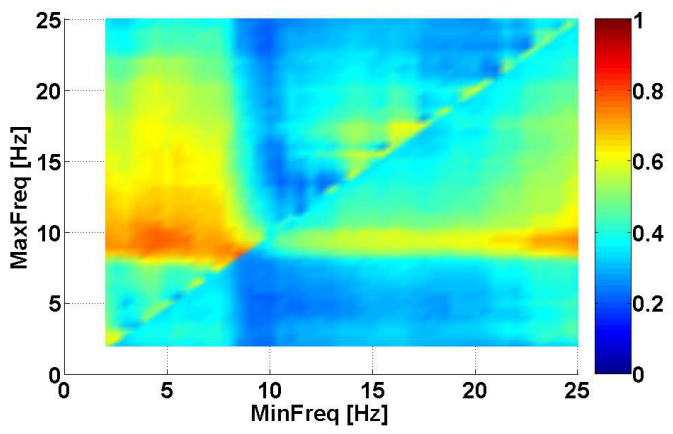

Figure 6: A diagram of $A U C$ values calculated for various frequency bands. In the upper left triangle of the diagram: the abscissa is the lower bound of the frequency band and the ordinate is the upper bound of the frequency band. In the lower right triangle of the diagram: the abscissa is the upper bound of the excluded frequency band and the ordinate is the lower bound of the excluded frequency band. The frequency varied from 2 to $25 \mathrm{~Hz}$ with the $0.5 \mathrm{~Hz}$ step. The background EEG was analyzed, the right hand tremor patients, the C3 cortex area.

central cortex area in the frequency bands $7.5-13.5 \mathrm{~Hz}(\mathrm{mu})$ and $18-24 \mathrm{~Hz}$ (beta-2) are independent. In other words, we have to demonstrate that the beta wave trains observed in the analysis are not the second harmonics of the mu wave trains. In this paper we demonstrate that these regularities in the mu and beta frequency bands are independent ones.

\section{Description of the Algorithm for Beta Wave Trains Elimination}

We have developed a special algorithm that eliminates from the analysis all beta wave trains in EEG signal that were observed simultaneously with the mu wave trains. There are four hypothetical cases when the beta wave trains can be observed simultaneously with the mu wave trains (superpositions of the mu wave train and the beta wave train):

1. The time interval of the beta wave train is located inside the time interval of the mu wave train (Figure 7). The duration of the beta wave train is smaller than the duration of the mu wave train.

2. The time interval of the mu wave train is located inside the time interval of the beta wave train (Figure 8). The duration of the beta wave train is bigger than the duration of the mu wave train.

3. The left end of the beta wave train is intersected with the right end of the mu wave train (Figure 9).

4. The right end of the beta wave train is intersected with the left end of the mu wave train (Figure 10).

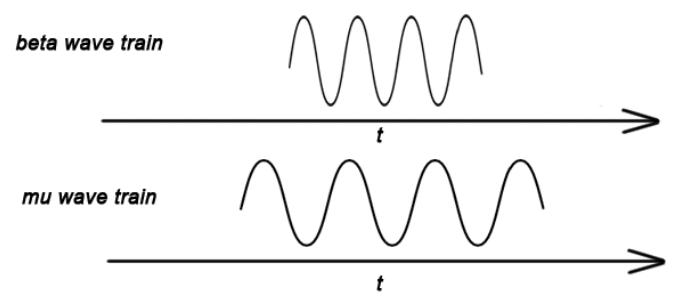

Figure 7: Superposition of a mu wave train and a beta wave train. The time interval of the beta wave train is located inside the time interval of the mu wave train.

The algorithm eliminates all beta wave trains that are intersected in time with any wave trains in the $2-14 \mathrm{~Hz}$ frequency range. Thus, all four cases are covered by this algorithm. In addition, the algorithm removes extra wave trains to exclude even the hypothetical possibility that the second or third harmonics of theta and mu EEG signals $(4-12 \mathrm{~Hz})$ are observed in the beta frequency range. This is done because EEG signals are not sine-shaped and the EEG wave train corresponds to a certain frequency band. Therefore, it is fundamentally impossible to predict exactly the frequency bands of the second and third harmonics of the wave trains under consideration.

Below we compare the results of EEG analysis with using and without using this algorithm of the wave train elimination. 


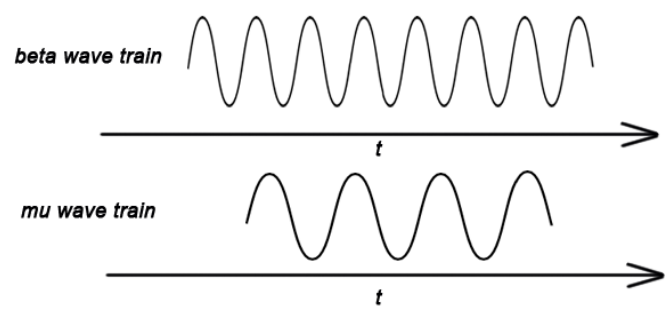

Figure 8: Superposition of a mu wave train and a beta wave train. The time interval of the mu wave train is located inside the time interval of the beta wave train.

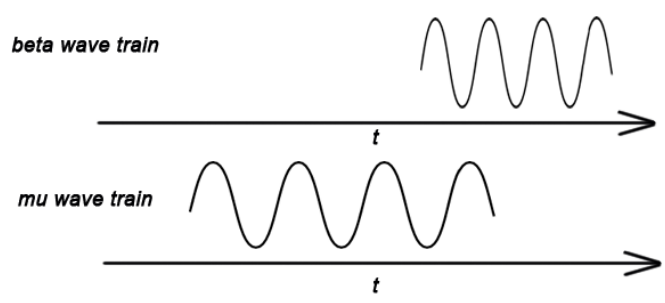

Figure 9: Superposition of a mu wave train and a beta wave train. The left end of the beta wave train is intersected with the right end of the mu wave train.

\section{The Experimental Setting}

We considered a set of EEG wave trains detected in a group of de novo Parkinson's disease patients and a healthy volunteer group. The group of patients included 17 patients with right hand tremor and 11 patients with left hand tremor in the first stage of Parkinson's disease without Parkinson's disease treatment. The group of healthy volunteers included 15 people.

The ages of the patients were from 38 to 71 years old; the mean age was 60 years old. The ages of the healthy volunteers were from 48 to 81 years old; the mean age was 58 years old. No statistically significant differences between the patients' ages and the volunteers' ages were detected. The amount of the male patients was 11; the amount of the female patients was 17 . The amount of the male healthy volunteers was 5 ; the amount of the female healthy volunteers was 10 . The size of the groups is typical for a neurophysiological examination.

The patients were clinically diagnosed according to the standard Hoehn and Yahr scale. All patients and volunteers were right-handed. A standard 10x20 EEG acquisition schema was used for the data collection. A background EEG was recorded in standard conditions. Examined person sat in an armchair relaxing with arms disposing on the armrests and fingers dangling freely from the ends of armrests. The eyes were closed during the recordings. A 41-channel digital EEG system Neuron-Spectrum-5 (Neurosoft Ltd.) was used. The sampling rate was $500 \mathrm{~Hz}$. The $0.5 \mathrm{~Hz}$ high-pass filter, the $35 \mathrm{~Hz}$ low-pass filter, and the $50 \mathrm{~Hz}$ notch filter were used. The duration of every record was about 3 minutes. The record was analyzed as is, without selection of areas in the signal.

In this paper, the $\mathrm{C} 3$ and $\mathrm{C} 4$ cortex areas are considered only, because these areas approximately correspond to the motor cortex areas and are situated in the scalp area that produces a minimal number of muscle artifacts.

\section{Results of the Analysis and Discussion}

Before applying the algorithm of the elimination of beta wave trains intersected with the mu wave trains, no statistically significant differences between quantities of wave trains in the right hand tremor patients and the healthy volunteers in both the $\mathrm{C} 3$ and $\mathrm{C} 4$ cortex areas were observed (see Figure 11). Also there were no significant differences between the left hand tremor patients and healthy volunteers (see Figure 12). Note that in [9] the Mann-Whitney test revealed significant differences between the data samples. This inconsistency of the results can be explained by the fact that another frequency range is considered in comparison with the previous work [9] (the $18-30 \mathrm{~Hz}$ frequency 


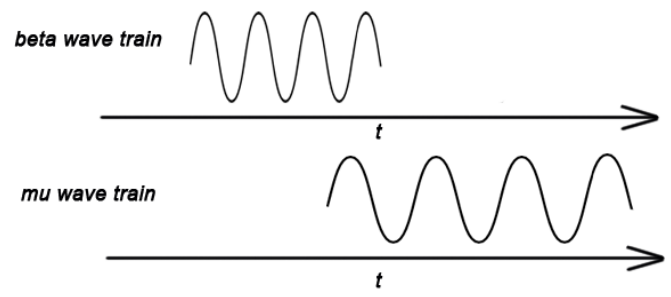

Figure 10: Superposition of a mu wave train and a beta wave train. The right end of the beta wave train is intersected with the left end of the mu wave train.

range is considered instead of the $12-25 \mathrm{~Hz}$ range). In addition, the right hand tremor patients and the left hand tremor patients are separated in present work. The algorithm of wave train detection also has been modified in comparison with the paper [9].
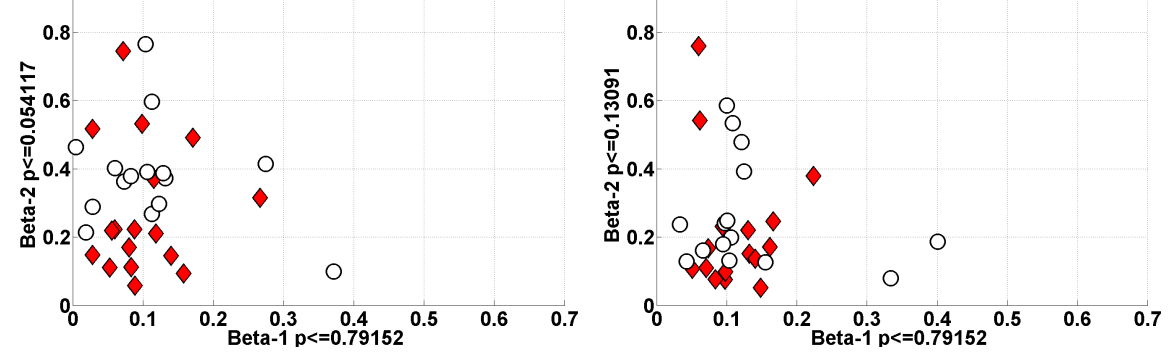

Figure 11: The scattering of the quantity of the beta wave trains in the right hand tremor patients and the healthy volunteers in the $\mathrm{C} 3$ cortex area (at the left) and in the $\mathrm{C} 4$ cortex area (at the right) before applying the algorithm of the elimination of beta wave trains intersected with the mu wave trains. There are no significant differences between the groups. The abscissa is the quantity of wave trains per second in the beta- 1 frequency band. The ordinate is the quantity of wave trains in the beta- 2 frequency band. The patients are indicated by diamonds and the healthy volunteers are indicated by circles.

After the processing of the experimental data by the wave trains elimination algorithm, the analysis of the data has confirmed that the beta band regularity does exist in its own. The statistically significant decrease of the beta wave train quantity was observed between the right hand tremor patients and the healthy volunteers in the $\mathrm{C} 3$ cortex area $(18-30 \mathrm{~Hz}$, Mann-Whitney, $p<0.02$, see Figure 13, left). Note that there is no significant difference in the C4 cortex area (see Figure 13, right), however, there was a statistical trend $(p<0.19)$. Thus, we have refined substantially the space and frequency localization of the investigated neurophysiological regularity in comparison with the paper [9].

The wave trains elimination algorithm decreases the quantity of beta- 2 wave trains in all groups (the right hand tremor patients, the left hand tremor patients, and the control group) by approximately $30 \%$. The fact that the statistically significant difference in the quantities of wave trains between the patients and the control group was detected by the Mann-Whitney test after the elimination of coincident mu and beta wave trains indicates that the eliminated wave trains are not a cause of the regularity. On the contrary, these wave trains were a noise that complicates the detection of the regularity.

It is interesting that a new neurophysiological regularity was discovered in the beta- 2 frequency band after applying the algorithm. The statistically significant differences of quantities of the beta- 2 wave trains were observed between the right hand tremor patients and the left hand tremor patients in both the C3 cortex area (Mann-Whitney, $p<0.03$, see Figure 14, left) and the C4 cortex area (Mann-Whitney, $p<0.02$, see Figure 14, right). The results of the test demonstrate that EEG of the right hand tremor patients and the left hand tremor patients are differed in both hemispheres. 

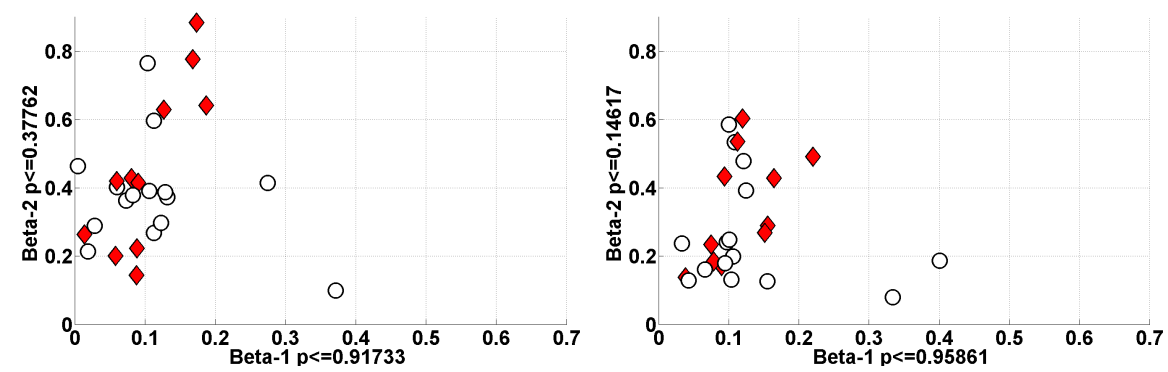

Figure 12: The scattering of the quantity of the beta wave trains in the left hand tremor patients and the healthy volunteers in the C3 cortex area (at the left) and in the C4 cortex area (at the right) before applying the algorithm of the elimination of beta wave trains intersected with the mu wave trains. There are no significant differences between the groups. The abscissa is the quantity of wave trains per second in the beta-1 frequency band. The ordinate is the quantity of wave trains in the beta- 2 frequency band. The patients are indicated by diamonds and the healthy volunteers are indicated by circles.
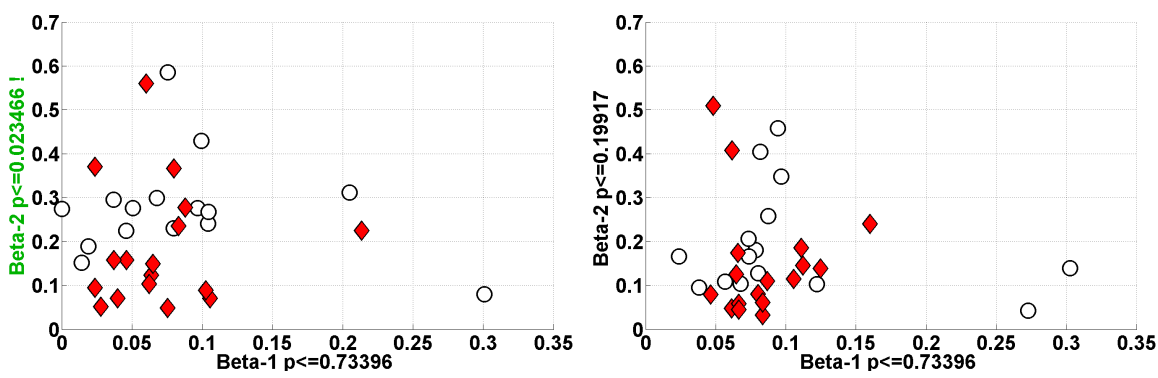

Figure 13: The scattering of the quantity of the beta wave trains in the right hand tremor patients and the healthy volunteers in the C3 cortex area (at the left) and in the $\mathrm{C} 4$ cortex area (at the right) after the processing the experimental data by the wave trains elimination algorithm. There is a significant difference between the groups at the left, but there is no significant difference between the groups at the right. The abscissa is the quantity of wave trains per second in the beta- 1 frequency band. The ordinate is the quantity of wave trains per second in the beta- 2 frequency band. The patients are indicated by diamonds and the healthy volunteers are indicated by circles.

\section{Conclusions}

A new method of signal processing and analysis for detailed investigation of time-frequency dynamics of the cortex electrical activity is developed. A distinctive feature of the method is a possibility to separate and analyze a specified set of wave trains in EEG signals. The results of the research give evidence that EEG analysis method based on the wave trains is prospective for:

- Looking for group statistical regularities in the early stages of Parkinson's disease that gives a basic knowledge about the disease and compensatory mechanisms in the brain cortex.

- Searching EEG features that are prospective for the early stages of Parkinson's disease diagnostics.

Using a special algorithm, we have demonstrated that a neurophysiological regularity discovered in the beta frequency band of EEG signals [9] is not an artifact caused by another regularity observed in the mu frequency band. In the course of the investigation, also, another neurophysiological regularity is discovered in the beta frequency band. The results of the analysis indicate that the right hand tremor patients and the left hand tremor patients differed in both hemispheres. This may be evidence that right hand tremor and left hand tremor Parkinson's disease patients must be diagnosed in different ways. Probably different approaches are necessary for treatment of the left hand tremor and right hand tremor patients as well. 

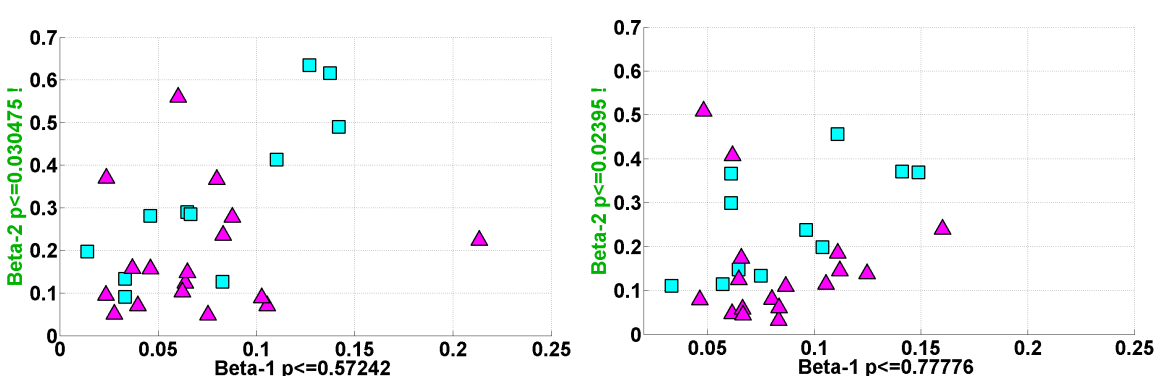

Figure 14: The scattering of the quantity of the beta wave trains in the right hand tremor patients and the left hand tremor patients in the C3 cortex area (at the left) and in the $\mathrm{C} 4$ cortex area (at the right) after the processing the experimental data by the wave trains elimination algorithm. There is a significant difference between the groups. The abscissa is the quantity of the wave trains per second in the beta- 1 frequency band. The ordinate is the quantity of the wave trains in the beta- 2 frequency band. The right hand tremor patients are indicated by triangles and the left hand tremor patients are indicated by squares.

\section{Acknowledgment}

Authors are grateful to Alexei V. Karabanov for selection and medical examination of the patients, to Galina D. Kuznetsova and Alexander F. Polupanov for co-operation and a help in the research, and Yuriy V. Obukhov and Mikhail N. Ustinin for a help in the statement of the problem. We acknowledge a partial financial support from the Russian Foundation for Basic Research, grant 15-07-07846.

\section{References}

[1] Lawhern V., Kerick S., Robbins K. A. Detecting alpha spindle events in EEG time series using adaptive autoregressive models // BMC Neuroscience. - 2013. - Vol. 14:101. http://www. biomedcentral.com/1471-2202/14/101.

[2] Determination of dominant simulated spindle frequency with different methods / E. Huupponen, W. D. Clercq, G. Gómez-Herrero et al. // Journal of Neuroscience Methods. — 2006. — Vol. 156. — Pp. 275-283.

[3] Sleep spindle detection through amplitude-frequency normal modelling / A. Nonclercq, C. Urbain, D. Verheulpen et al. // Journal of Neuroscience Methods. — 2013. - Vol. 214. - Pp. 192-203.

[4] Improved spindle detection through intuitive pre-processing of electroencephalogram / A. Jaleel, B. Ahmed, R. Tafreshi et al. // Journal of Neuroscience Methods. — 2014. - Vol. 233. — Pp. 1-12.

[5] Camilleri T. A., Camilleri K. P., Fabri S. G. Automatic detection of spindles and K-complexes in sleep EEG using switching multiple models // Biomedical Signal Processing and Control. — 2014. - Vol. 10. - Pp. 117-127.

[6] Sleep spindle detection using time-frequency sparsity / A. Parekh, I. Selesnick, D. Rapoport, I. Ayappa // IEEE Signal Processing in Medicine and Biology Symposium. — Philadelphia, PA: IEEE, 2014. - Pp. 1-6.

[7] O'Reilly C., Nielsen T. Automatic sleep spindle detection: benchmarking with fine temporal resolution using open science tools // Frontiers in Human Neuroscience. - 2015. - Vol. 9:353. http://doi .org/10.3389/fnhum.2015.00353.

[8] Obukhov Y., Korolyov M., Gabova A. et al. Patent No 2484766 Rossiskaya Federacia. Sposob rannei electroencephalographicheskoi diagnostiki bolezni Parkinsona. 20.06.2013. - 2013. - In Russian.

[9] Sushkova O., Morozov A., Gabova A. A method of analysis of EEG wave trains in early stages of Parkinson's disease // International Conference on Bioinformatics and Systems Biology (BSB-2016) / IEEE. — 2016. — Pp. 1-4.

[10] Sushkova O. S., Morozov A. A., Gabova A. V. Development of a method of analysis of EEG wave packets in early stages of Parkinson's disease // Proceedings of the International conference Information Technology and Nanotechnology (ITNT 2016, Samara, Russia, May 17-19, 2016). Samara: CEUR, 2016. - Pp. 681-690. http://ceur-ws .org/Vol-1638/Paper82.pdf.

[11] Data mining in EEG wave trains in early stages of Parkinson's disease / O. S. Sushkova, A. A. Morozov, A. V. Gabova, A. V. Karabanov // Proceedings of the 12th Russian-German Conference on Biomedical Engineering (RGC XII, Suzdal, July 4-7 2016). - Suzdal: Vladimir State University, 2016. - Pp. 80-84.

[12] Neural mass models describing possible origin of the excessive beta oscillations correlated with Parkinsonian state / C. Liu, Y. Zhu, F. Liu et al. // Neural Networks. - 2017. - Vol. 88. - Pp. 65-73.

[13] Pavlides A., Hogan S. J., Bogacz R. Computational models describing possible mechanisms for generation of excessive beta oscillations in Parkinson's disease // PLOS Computational Biology. — 2015. — no. 12. - Pp. 1-29.

[14] Kapitsa I., Nerobkova L., Voronina T. EEG correlates of an early stage of a Parkinson illness in experiment on mice of the strain C57BL/6 // Biomeditsina. - 2014. - no. 1. - Pp. 54-60. - In Russian.

[15] Resting state oscillatory brain dynamics in Parkinson's disease: An MEG study / J. Bosboom, D. Stoffers, C. Stam et al. // Clinical Neurophysiology. - 2006. - Vol. 117. — Pp. 2521-2531. 